Voix et Images

\title{
Dany Laferrière : la traversée du continent intérieur
}

\section{Jean Morency et Jimmy Thibeault}

Volume 36, numéro 2 (107), hiver 2011

Dany Laferrière

URI : https://id.erudit.org/iderudit/1002438ar

Aller au sommaire du numéro

Éditeur(s)

Université du Québec à Montréal

ISSN

0318-9201 (imprimé)

1705-933X (numérique)

Découvrir la revue

Citer ce document

Morency, J. \& Thibeault, J. (2011). Dany Laferrière : la traversée du continent intérieur. Voix et Images, 36(2), 7-13. d'utilisation que vous pouvez consulter en ligne.

https://apropos.erudit.org/fr/usagers/politique-dutilisation/ 


\title{
DANY LAFERRIÈRE： LA TRAVERSÉE DU CONTINENT INTÉRIEUR
}

\author{
$+++$ \\ JEAN MORENCY \\ Université de Moncton \\ JIMMY THIBEAULT \\ Université de Moncton
}

Depuis 1985, Dany Laferrière a publié dix-huit livres - surtout des romans, mais aussi des essais et des livres pour la jeunesse - qui ont été traduits en plusieurs langues. En plus d'avoir mené une carrière littéraire prolifique, marquée par l'obtention de nombreux prix, il a également touché au cinéma en contribuant à la scénarisation de quelques-uns de ses livres et en réalisant, en 2005, un film qui s'inscrit dans la continuité de son œuvre romanesque (Comment conquérir l'Amérique en une nuit, primé au Festival des films du monde de Montréal). Pourtant, Dany Laferrière a d'abord été perçu comme un personnage public, médiatique, dont les interventions à la télévision, à la radio et dans les journaux se sont caractérisées à la fois par l'humour, la justesse et la profondeur du regard porté sur le monde. Tout se passe comme si, depuis la parution de son premier roman, la figure de l'écrivain s'était effacée derrière celle, plus flamboyante, du chroniqueur. On constate aussi que, pour parler de l'écrivain, les médias ont essentiellement recouru, du moins jusqu'à tout récemment, à l'image de l'auteur provocateur suscitée par la parution de Comment faire l'amour avec un Nègre sans se fatiguer ou à celle de l'auteur haïtien qui, dans L'odeur du café, raconte avec nostalgie son enfance à Petit-Goâve. Deux images figées d'un écrivain qui, ironiquement, a consacré une partie de son travail à briser les lieux communs de l'imaginaire collectif. En fait, Dany Laferrière semble surtout se définir, ainsi qu'il le souligne lui-même avec beaucoup d'humour dans ses romans, particulièrement dans Cette grenade dans la main du jeune Nègre est-elle une arme ou un fruit? et dans Je suis un écrivain japonais, comme un grand titreur, c'est-à-dire un auteur dont la force d'évocation des titres et l'image médiatique suffisent à créer chez le lecteur l'illusion de saisir l'essentiel du personnage et de l'œuvre. Cette réception de l'œuvre tend cependant à changer depuis l'attribution, en 2009 et 2010, de nombreux prix pour son roman L'énigme du retour — dont le prix Médicis, le Grand Prix du livre de Montréal, le Prix des libraires - sans compter la nomination de l'écrivain au rang de personnalité de l'année 2009 par La Presse/Radio-Canada et l'attribution du Grand Prix littéraire international du festival Metropolis bleu de Montréal à l'ensemble de son œuvre. Le public découvre donc Dany Laferrière, l'écrivain, qui d'une certaine manière, transcende le personnage médiatique et le titreur. 
Si l'image populaire de l'auteur provient surtout du retentissement de quelques titres et d'interventions ponctuelles dans les médias, il ne faut pas croire que les romans qui ont suivi Comment faire l'amour... sont passés inaperçus auprès de la critique universitaire. Bien que l'œuvre de Dany Laferrière n'ait pas fait, à ce jour, l'objet d'un ouvrage collectif ou d'un dossier dans une revue universitaire, elle est étudiée non seulement au Québec, mais aussi en Amérique et en Europe. Il est d'ailleurs intéressant de noter que la première étude d'envergure portant sur l'ensemble de l'œuvre romanesque de l'auteur nous vient d'Autriche ${ }^{1}$ et qu'un bon nombre d'articles traitant de ses romans sont rédigés dans une autre langue que le français, surtout en anglais. Ainsi, plusieurs chercheurs, du Québec et d'ailleurs, ont souligné l'intérêt que présentent les romans de Laferrière, notamment pour la réflexion, très actuelle, sur les rapports à l'espace dans le contexte de migration, d'ouverture à l'autre et d'hybridation qu'impliquent des frontières culturelles de plus en plus poreuses. Chez Laferrière, il y a effectivement une reconfiguration de l'espace identitaire qui passe principalement par une "mise à nu» des stéréotypes, par le refus des lieux communs, par le désir, en fait, de dépasser les déterminismes de la naissance, ce qui permet au sujet qui se raconte de se reconnaître à partir des expériences personnelles qui le définissent dans l'ici et le maintenant. On remarque donc l'apport important de l'œuvre de Dany Laferrière, resté fidèle à l'espace littéraire du Québec, dans la reconfiguration de l'imaginaire québécois depuis les années 1980.

\section{L'ESPACE CONTINENTAL DE L'EUVRE}

Le premier roman de Dany Laferrière, Comment faire l'amour..., paraît à un moment où le roman québécois est en période de renouvellement: depuis les années 1970, la problématique identitaire nationale est lentement délaissée au profit d'une réflexion sur le devenir des individus qui habitent l'espace américain. Au «nous» bien défini du discours national s'oppose désormais, avec plus d'insistance dans les années 1980, un «soi» en quête de sens. Ce dernier cherche à se définir dans le monde à partir des traces de son américanité. Ce nouveau «soi» décentré vise ainsi l'expression d'une certaine «Franco-Amérique» - telle que l'ont décrite les géographes Dean Louder, Jean Morisset et Éric Waddell ${ }^{2}$ - qui existe non seulement en dehors des frontières du Québec, mais aussi dans la conscience des sujets qui l'explorent, de telle sorte qu'il nous est donné d'assister à un brouillage des codes littéraires traditionnels et à une référence plus assumée aux écrivains américains. L'œuvre de Dany Laferrière participe de ce mouvement qui conduit plusieurs écrivains québécois à se réclamer du grand roman américain en s'identifiant, d'une part, à la culture littéraire américaine et en

$$
+++
$$

1 Ursula Mathis-Moser, Dany Laferrière. La dérive américaine, Montréal, VLB éditeur, coll. «Les champs de la culture», 2003, 344 p. 2 Voir Dean Louder et Éric Waddell (dir.), Du continent perdu à l'archipel retrouvé. Le Québec et l'Amérique française, Québec, Presses de l'Université Laval, coll. «Travaux du Département de géographie de l'Université Laval», 1983, 292 p.; Dean Louder, Jean Morisset et Éric Waddell (dir.), Vision et visages de la Franco-Amérique, Québec, Septentrion, 2001, 346 p.; Dean Louder et Éric Waddell (dir.), Franco-Amérique, Québec, Septentrion, 2008, 373 p. 
situant, d'autre part, son parcours, depuis Petit-Goâve jusqu'à Montréal, dans l'expression d'une Franco-Amérique associée non seulement au Québec, mais aussi à Haïti, une Haïti moderne, américaine - au sens continental du terme. On n'a qu'à penser, par exemple, à toute l'énergie qu'on retrouve dans les romans dont l'action se déroule à Port-au-Prince, notamment La chair du maître et Le goût des jeunes filles, où des personnages affirment leur rêve d'émancipation, de pouvoir et de succès. Celuici n'est pas sans rappeler le rêve américain tel que représenté dans Comment faire l'amour... et Cette grenade...

Comme plusieurs romanciers québécois de sa génération, qui explorent le rêve américain et le rapport que le Québec entretient avec lui, Laferrière reconnaît l'influence américaine dans son œuvre, influence qui se remarque par des références soutenues aux grands écrivains américains et, particulièrement, afro-américains. Il se démarque cependant de ses contemporains, comme le note Anne Marie Miraglia, en ceci que

le mythe du rêve américain est formulé, pour la première fois, par un Noir immigré au Québec. Ce fait a des conséquences importantes pour la représentation de l'Amérique et de l'identité culturelle américaine, et explique aussi la place toute spéciale accordée aux écrivains afro-américains Chester Himes et James Baldwin ${ }^{3}$.

Ainsi, Laferrière intègre à l'américanité québécoise un nouveau paradigme qui aura pour effet d'élargir la réflexion continentale: l'exploration du continent ne se fait plus à la recherche de traces originelles de la présence canadienne-française en Amérique (celles des pionniers et découvreurs que suivent, par exemple, Jack Waterman et Pitsémine dans Volkswagen blues ${ }^{4}$ ); elle touche davantage à la reconnaissance et à l'inscription du soi dans une expérience continentale avant tout définie au présent.

Cette expérience continentale, Dany Laferrière avoue d'ailleurs la vivre luimême au plus profond de son être; elle s'avère donc intimement liée à son parcours entre Port-au-Prince, Montréal et Miami. L'écrivain affirme, par exemple, qu'il est «né physiquement en Haïti mais comme écrivain à Montréal ${ }^{5}$ ». Il avance même qu'il habite littéralement tous les lieux qui servent à le définir : « J'ai l'habitude de dire que je suis un homme en trois morceaux: mon cœur est à Port-au-Prince, mon esprit à Montréal, et mon corps à Miami ${ }^{6}$.» Ailleurs, Laferrière précise: "On parle toujours de moi en termes de territoire. Or, je ne me sens pas Haïtien ni Québécois. Plutôt Américain ${ }^{7}$. » Ce qui amène Ursula Mathis-Moser à constater que

si les critiques s'obstinent à considérer Dany comme «un des auteurs les plus captivants de la francophonie», "un des plus importants écrivains [...] de Montréal et

$$
+++
$$

3 Anne Marie Miraglia, "Dany Laferrière, l'identité culturelle et l'intertexte afro-américain », Présence francophone, n 54, 2000, p. 125-126. 4 Jacques Poulin, Volkswagen blues, Montréal Québec Amérique, coll. «Littérature d'Amérique», 1984, 290 p. 5 Hélène Marcotte, «Interview : Je suis né comme écrivain à Montréal », Québec français, n 79, automne 1990, p. 81. 6 Dany Laferrière, J'écris comme je vis. Entretien avec Bernard Magnier, Outremont, Lanctôt, 2000, p. 59. 7 Francine Bordeleau, «Dany Laferrière sans arme et dangereux. Entrevue», Lettres québécoises, n 73, printemps 1994, p. 10. 
d'Haïti» ou bien comme un des «meilleurs romanciers dits haïtiens de la diaspora ", il faudra encore se demander si Dany n'est pas aussi l'un des meilleurs écrivains américains «à qui il est arrivé tout simplement d'écrire en français » ${ }^{8}$.

Dès lors, l'américanité de son œuvre romanesque ne doit plus être lue uniquement à travers les romans qui mettent en scène l'espace nord-américain, dont les plus cités sont évidemment Comment faire l'amour... et Cette grenade..., mais aussi comme une expérience inhérente à l'ensemble de l'œuvre. En fait, l'écriture de Dany Laferrière propose une pénétration de l'Amérique, voire de la Franco-Amérique, qui passerait désormais par les deux pôles que sont Haïti et le Québec.

\section{L'ESPACE DU SOI}

Cette pénétration du continent, ce regard que porte le narrateur sur l'Amérique, se présente dans un cycle romanesque qui devient un espace de réinterprétation et d'affirmation du soi. La notion même d' «Autobiographie américaine», qui rassemble les dix premiers romans de Dany Laferrière - de Comment faire l'amour... au Cri des oiseaux fous - et à la suite desquels s'inscrivent Je suis un écrivain japonais ainsi que L'énigme du retour, sous-tend d'ailleurs l'importance que revêt, dans le récit, la subjectivité du narrateur qui interprète le monde qu'il habite. Le récit «autobiographique» chez Laferrière sert moins à se regarder vivre dans un passé recomposé qu'à porter un regard critique sur le monde qui a façonné l'homme qu'il est devenu ainsi que sur celui qui l'entoure au présent, car les romans tournent toujours autour d'un dialogue entre la posture du narrateur/écrivain et l'univers de son récit. On constate d'ailleurs cette mise à distance du discours narratif avec l'univers du récit dès l'ouverture de L'odeur du café, alors que le narrateur se pose nettement comme un observateur d'une autre époque:

\section{L'ÉTÉ 63}

J'ai passé mon enfance à Petit-Goâve, à quelques kilomètres de Port-au-Prince. Si vous prenez la Nationale Sud, c'est un peu après le terrible morne Tapion. Laissez rouler votre camion (on voyage en camion bien sûr) jusqu'aux casernes (jaune feu), tournez tranquillement à gauche, une légère pente à grimper, et essayez de vous arrêter au 88 de la rue Lamarre.

Il est fort possible que vous voyiez, assis sur la galerie, une vieille dame au visage serein et souriant à côté d'un petit garçon de dix ans. La vieille dame, c'est ma grandmère. Il faut l'appeler Da. Da tout court. L'enfant, c'est moi. Et c'est l'été $63^{9}$.

Ce resserrement du champ narratif suggère bien ce regard autre, au sens temporel et géographique, qui s'intègre lentement à l'espace où se déroule l'action afin de mieux observer le monde, rôle qui incombe à Vieux Os, alter ego de l'auteur. Un processus

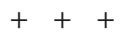

8 Ursula Mathis-Moser, Dany Laferrière. La dérive américaine, p. 38. 9 Dany Laferrière, L'odeur du café, Montréal, TYPO, 1999 [1991], p. 13. 
narratif qui se poursuit dans Le charme des après-midi sans fin, que la narration inscrit dans la suite de L'odeur du café. On retrouve également le même phénomène dans La chair du maître, qui s'ouvre sur un chapitre intitulé «Pour planter le décor ${ }^{10}$ » dont le rôle premier semble être de mettre en contexte l'ensemble du livre - mais aussi l'ensemble des romans dont l'action se situe à Port-au-Prince - à la fois dans le parcours du narrateur qui se raconte et par rapport à un point de vue qui est celui de la relecture, au présent, du passé trouble d'Haïti :

\begin{abstract}
Tout a commencé, il y a très longtemps, j'avais à peine quinze ans. C'était en 1968, l'année de tous les bouleversements. À l'époque, j'étais profondément troublé par ce courant électrique qui me traversait littéralement le corps à la vue de toute fille. Ce n'était plus le temps du sentiment idéal (mon amour fou pour Vava), mais celui de tous les désirs. Disons-le net: du sexe [...] Malheureusement, aujourd'hui, à quarantequatre ans, je peux dire que l'affaire est encore au même point. La seule différence c'est que si, en 1968, j'ignorais la raison de cette fièvre, je commence, à mi-vie, à entrevoir vaguement une tentative d'explication: si le sexe est sain, il n'est pas innocent ${ }^{11}$.
\end{abstract}

C'est à partir de cette impression intime d'un courant électrique et par la reconnaissance, après coup, du sens érotique de ce courant que s'inscrivent les récits singuliers des différents personnages qui habitent La chair du maître et qui sont aux prises avec l'urgent besoin de vivre et de survivre dans un monde de pouvoir et de guerre: «Il n'y a pas d'avenir. Tout se passe au présent. À l'instant même. Et tous les coups sont permis. Et donnés. C'est la guerre ${ }^{12}$ !» Encore une fois, le sujet du récit, le personnage-narrateur faisant figure de «soi autobiographique», devient prétexte à l'observation après coup, au regard que porte le narrateur/auteur sur un espace social qu'il a habité et qui, comme le besoin de raconter tend à le montrer, l'habite.

Laferrière propose donc une interprétation libre de la notion $\mathrm{d}^{\prime}$ « autobiographie» en posant ses romans à la frontière de la réalité et de la fiction, ce qui a pour effet de mettre les émotions qu'évoquent les lieux habités au centre du récit, plutôt que la sèche énumération des faits réels. Ursula Mathis-Moser a d'ailleurs bien montré, au sujet de la véracité des événements dits biographiques, que si Dany Laferrière affirme volontiers qu'il se pose lui-même comme le seul véritable sujet de son écriture, il précise que «dans ses livres tout se passe entre le mensonge et la vérité ${ }^{13}$ ». Pour Laferrière, la frontière entre la fiction et la réalité, entre le mensonge et la vérité, n'est pas stable et place l'auteur dans une double posture qui fait de l'«Autobiographie américaine» un récit appartenant à la fois au domaine de l'imaginaire et à celui du réel.

Ici, le narrateur reste donc en contrôle du discours, de la représentation qu'il donne du monde, en se posant lui-même au centre de toute interprétation : il utilise le stéréotype de la Blonde, dans Comment faire l'amour..., afin de briser celui du Nègre; il pose son discours identitaire en opposition à celui que tiennent les groupes

10 Ce chapitre a été coupé dans la réédition du livre sous le titre Vers le sud. 11 Dany Laferrière, La chair du maître, Outremont, Lanctôt, 1997, p. 11. 12 Ibid., p. 18. 13 Ursula Mathis Moser, Dany Laferrière. La dérive américaine, p. 51. 
raciaux qu'il rencontre dans Cette grenade... ; il raconte l'univers de Petit-Goâve depuis la galerie de sa grand-mère dans L'odeur du café; il se réclame, enfin, du droit de se dire "écrivain japonais» dans Je suis un écrivain japonais. Encore une fois, dans cette représentation «autobiographique» du monde, c'est le soi qui se dit et qui cherche à confirmer la place qu'il y occupe. Une quête qui, dans une certaine mesure, trouve son aboutissement dans L'énigme du retour, où le narrateur va au-delà des espaces d'appartenance, le Nord, le Sud, pour atteindre l'essence même du soi. Ce parcours intime est décrit, dans ce dernier roman, par un mouvement de circularité qui amène le narrateur à se retrouver dans le lieu du rêve et de l'enfance: «On me vit aussi sourire/dans mon sommeil./Comme l'enfant que je fus/du temps heureux de ma grand-mère./Un temps enfin revenu./C'est la fin du voyage ${ }^{14}$. »

Le présent dossier se donne pour objectif de réunir des textes qui permettront aux lecteurs de découvrir, par des lectures inédites de quelques-uns des romans qui constituent l'univers narratif de Dany Laferrière, cet espace du soi, ce discours sur le monde perçu à travers la subjectivité de celui qui tente d'affirmer son individualité. Les articles que nous avons réunis explorent principalement l'espace de l'affirmation du soi dans le contexte de la mise en écriture ou, pour reprendre l'expression de Laferrière lui-même, de la venue au monde de l'auteur comme écrivain. Il nous a semblé pertinent de mettre en lumière la réflexion identitaire que les romans du cycle nord-américain mettent en scène à travers un double rapport au monde de l'immigrant. D'une part, confronté au regard de l'autre, il porte lui-même un regard autre sur l'espace habité ; d'autre part, son rapport au lieu de l'origine, qu'il sent de plus en plus éloigné dans l'espace, mais surtout dans le temps, l'amène à s'interroger sur sa propre présence au monde ainsi que sur le véritable sens de se dire soi. Ce double rapport au monde est particulièrement important et intéressant à explorer puisqu'il détermine, nous l'avons déjà souligné, le regard porté sur le lieu de l'origine dans les récits autobiographiques de l'enfance et de l'adolescence, mais également la relation qui s'établit au présent, après trente années d'absence, entre la narration et Haïti. Un double rapport au monde, finalement, qui se construit au fil d'une longue traversée du continent intérieur.

Le dossier s'ouvre sur un entretien avec Dany Laferrière réalisé le $1^{\text {er }}$ décembre 2009, soit trois semaines après l'attribution du prix Médicis à L'énigme du retour et un mois et demi avant le tremblement de terre qui a secoué Haïti, le 21 janvier $2010^{15}$; cet entretien permet à l'écrivain de revisiter son œuvre littéraire, notamment à la lumière de son dernier roman. Le premier article du dossier, signé par Jimmy Thibeault, s'inscrit dans ce mouvement d'ensemble en montrant comment l'œuvre de Laferrière, et particulièrement son «Autobiographie américaine», correspond en fait à un vaste projet d'individualité qui amène l'écrivain à rejeter toutes les étiquettes et à faire des expériences intimes les seules références identitaires qui importent véritablement. Dans son article judicieusement intitulé «Un Japon de papier », Alain Farah montre bien comment ce projet trouve son aboutissement dans Je suis un écrivain

14 Dany Laferrière, L'énigme du retour, Montréal, Boréal, 2009, p. 286. 15 On sait que cette catastrophe a failli emporter l'écrivain et l'a poussé à écrire Tout bouge autour de moi, une chronique des événements publiée en mars 2010 chez Mémoire d'encrier. 
japonais: en s'appuyant sur la notion d'œuvre incomparable, l'auteur analyse la manière dont Laferrière parvient, dans ce roman, à décloisonner les genres littéraires et à occuper du même coup l'écriture, en construisant une «expérience de pensée » à nulle autre pareille, fondée sur l'établissement de correspondances inédites et sur l'usage qui est fait des clichés collectifs.

Lori Saint-Martin aborde elle aussi, mais sous un angle différent, la question des clichés dans son article consacré à Comment faire l'amour avec un Nègre sans se fatiguer. Elle y analyse la représentation des personnages féminins en s'interrogeant sur le recours paradoxal qui est fait, dans le roman, à un discours sexiste dans le but de dénoncer le racisme. Saint-Martin montre bien que la contestation des stéréotypes raciaux et sexuels dans ce roman n'est pas exempte de contradictions, bien au contraire. En cela l'écrivain ne semble remettre en question que les stéréotypes raciaux, se contentant de reproduire une vision convenue, pour ne pas dire sexiste, des femmes.

Cette question des stéréotypes dans l'œuvre de Dany Laferrière constitue aussi le point de départ de l'article d'Ursula Mathis-Moser. Celle-ci met l'accent sur le rejet des stéréotypes nationaux et du nationalisme culturel dans Je suis un écrivain japonais, rejet qui s'appuie sur la valorisation de l'écrivain et de la position qu'il occupe dans un espace de l'entre-deux révélateur de la «littérature-monde» actuelle, laquelle se situe dans le prolongement de la Weltliteratur imaginée par Goethe au xIXe siècle.

L'importance des clichés et des stéréotypes ne doit toutefois pas nous faire oublier que l'œuvre de Dany Laferrière reste empreinte de gravité, comme le montre bien l'article d'Anne Marie Miraglia, qui propose une lecture comparée de Pays sans chapeau et de L'énigme du retour, lecture fondée sur la thématique du retour et de la mort dans les deux romans. Le retour au pays natal s'accompagne en effet d'une réflexion sur la mort, que ce soit celle de Da, la grand-mère adorée, ou celle de ce père à qui l'écrivain n'aura parlé qu'une seule fois. Miraglia montre bien la profondeur de cette thématique, notamment par l'entremise des liens féconds qu'elle instaure avec le Cahier d'un retour au pays natal d'Aimé Césaire.

Pour terminer, la bibliographie préparée par Mathieu Lanteigne rend compte du succès des romans de Dany Laferrière et de l'intérêt de indéniable que lui porte la critique, autant journalistique qu'universitaire. Nous espérons que les différents textes composant le présent dossier pourront contribuer à la vitalité de ce discours critique ${ }^{16}$.

$$
++
$$

16 Afin d'alléger le système de notes, les références aux œuvres de Dany Laferrière mentionnées dans ce dossier seront indiquées par des sigles, suivis du folio et placés entre parenthèses dans le texte: Comment faire l'amour avec un Nègre sans se fatiguer (CFA), Montréal, TYPO, 2002 [1985], 192 p. ; Éroshima (É), Montréal, TYPO, 1998 [1987], 160 p. ; L'odeur du café (OC), Montréal, TYPO, 1999 [1991], 240 p. ; Le goût des jeunes filles (GJF), Montréal, VLB éditeur, 2004 [1992], 331 p.; Cette grenade dans la main du jeune Nègre est-elle une arme ou un fruit? (CG), Montréal, VLB éditeur, 2002 [1993], 353 p.; Chronique de la dérive douce (CDD), Montréal, VLB éditeur, 1994, 136 p. ; Pays sans chapeau (PSC), Outremont, Lanctôt, 1996, 221 p. ; La chair du maître (CM), Outremont, Lanctôt, 1997, 311 p. ; Le charme des après-midi sans fin (CAF), Outremont, Lanctôt, 2001 [1997], 223 p. ; Le cri des oiseaux fous (COF), Outremont, Lanctôt, 2000, 318 p.; J'écris comme je vis. Entretien avec Bernard Magnier (JÉ), Outremont, Lanctôt, 2000, 247 p.; Je suis fatigué (JSF), Montréal, TYPO, 2005 [2000], 232 p.; Vers le sud (VS), Montréal, Boréal, 2006, 256 p. ; Je suis un écrivain japonais (ÉJ), Montréal, Boréal, 2008, 272 p. ; L'énigme du retour (ÉR), Montréal, Boréal, 2009, 296 p.; Tout bouge autour de moi (TBAM), Montréal, Mémoire d'encrier, coll. «Chroniques», 2010, $159 \mathrm{p}$. 